\title{
Prolongation of biologic dosing intervals in patients with stable psoriasis: a
}

\section{feasibility study}

van Bezooijen, Ji S., MD ${ }^{1,2}$; van Doorn, Martijn B.A., MD, PhD²; Schreurs, Marco W.J., PhD ${ }^{3}$; Koch, Birgit C.P., PharmD ${ }^{1}$; Henk te Velthuis, $\mathrm{PhD}^{4}$; Prens, Errol P., MD, PhD ${ }^{2,3} *$; van Gelder, Teun, $\mathrm{MD}, \mathrm{PhD}^{1} *$

Hospital Pharmacy ${ }^{1}$; Departments of Dermatology ${ }^{2}$; and Immunology ${ }^{3}$; Erasmus MC, University Medical Center Rotterdam, The Netherlands, Department of R\&D, Sanquin Reagents ${ }^{4}$; Department of Immunopathology, Sanquin Research and Landsteiner Laboratory, Academic Medical Center, Amsterdam, The Netherlands

*Both authors contributed equally

Word count: 3543 words, abstract, 28 references, 2 Figures and 1 table.

Potential conflicts of interest: M.B.A. van Doorn has acted as a consultant for Abbott, Janssen, LEO Pharma, and Pfizer and has been an investigator for Eli Lilly, Idera Pharmaceuticals, and Novartis. M. Schreurs has received financial compensation for seminars from Biognost, Inova, and Thermo Scientific. B. Koch has acted as a consultant for Pfizer. Henk te Velthuis is an employee of Sanquin Research and Landsteiner Laboratory, the manufacturer of the used ELISA kits. E. Prens has acted as a consultant for AbbVie, Amgen, Astra-Zeneca, Baxter, Eli Lilly, Galderma, Jansse, n-Cilag, Novartis, and Pfizer and has received investigator-initiated research grants from Pfizer, Janssen-Cilag, and AbbVie. T. van Gelder has been on the speakers' bureau or worked as consultant for Sandoz, Novartis, Teva, Chiesi, Astellas, and Roche. For the remaining authors, no conflicts of interest were declared. 


\section{Financial support: none}

Correspondence: J.S. van Bezooijen, Department of Dermatology and Hospital Pharmacy, Erasmus MC, P.O. Box 2040, 3000 CA Rotterdam, the Netherlands (j.vanbezooijen@erasmusmc.nl; phone: $+31633048922)$.

\section{Abstract}

Background: Biologics are usually licensed according to the "one dose fits all" principle. It is therefore suspected that a significant number of psoriasis patients are overtreated. However, evidence for successful dose reduction of biologics in psoriasis is scarce. The aim of this study was to investigate whether the dosing interval of three biologics, adalimumab, etanercept, or ustekinumab could be prolonged successfully in patients with plaque psoriasis.

\section{Methods}

In a prospective exploratory cohort study, 59 psoriasis patients on maintenance treatment with adalimumab, etanercept, or ustekinumab were included. After a run-in period of six weeks, the dosing interval of the biologics was prolonged according to a predefined schedule. Our primary objective was to determine the proportion of patients that could maintain a successful prolongation of the per label dosing interval. Secondary objectives were to evaluate the predictive value of baseline serum trough concentrations for successful dosing interval prolongation and to explore the feasibility of dosing interval prolongations in offlabel treated patients.

\section{Results}

In the per label group, 7 out of $16(44 \%)$ adalimumab patients, 5 out of $16(31 \%)$ etanercept patients, 2 out of $10(20 \%)$ ustekinumab patients achieved a successful dosing interval prolongation. Baseline serum trough concentrations did not differ significantly between patients with successful dosing interval prolongation and 
failures. In the off-label group, prolongation in patients with already extended intervals was unsuccessful. For patients with shortened intervals, minor prolongation was successful in 3 out of 17 (17.6\%) patients.

\section{Conclusion}

Prolongation of the per label biologic dosing interval was feasible in approximately $30 \%$ of psoriasis patients with stable minimal disease activity and can reduce costs in clinical practice. Baseline serum trough concentrations were not predictive for successful dosing interval prolongation.

Keywords: biologics, adalimumab, etanercept, ustekinumab, dosing interval prolongation, psoriasis

\section{Background}

Biologics are effective drugs for the treatment of moderate to severe plaque psoriasis. As a result, their use is increasing and many new biologics and biosimilars are being developed. Biologics are usually licensed and marketed according to the "one dose fits all" principle, and dose regimens with fixed dosing intervals are recommended. However, a number of studies in patients with stable rheumatoid arthritis (RA) have shown that the per label biologic dose can be reduced without a subsequent increase in disease activity (1-3). Recently, biologic dose reduction and dosing interval prolongation have also been described in patients with psoriasis $(4,5)$.

Measurement of high trough concentrations supports these dose reductions in RA, since adalimumab levels above $8 \mu \mathrm{g} / \mathrm{mL}$ in RA patients were found to have no clear additional beneficial effect on disease activity (6). Similar findings were reported in psoriasis where one-third (44 out of 135) of psoriasis patients had adalimumab concentrations above $7 \mu \mathrm{g} / \mathrm{mL}$ without further increase in clinical improvement (7). Chen et al. also showed that after 24 weeks of adalimumab dose reduction with $50 \%$, the trough concentrations above the cutoff value of $6.4 \mu \mathrm{g} / \mathrm{mL}$ predicted persistent remission in patients with RA (3). The adalimumab trough concentrations were significantly higher in RA patients with persistent remission (median $10.5 \mu \mathrm{g} / \mathrm{mL}$ ) or 
with low disease activity $(4.5 \mu \mathrm{g} / \mathrm{mL})$ than in those with disease flare $(0.9 \mu \mathrm{g} / \mathrm{mL})$. These data suggest that a substantial proportion of patients on biologics are indeed overtreated. In view of these studies in RA and psoriasis, we hypothesized that biologic trough concentrations could be a useful indicator for dose reduction of adalimumab and possibly other biologics that are currently being used for patients with psoriasis.

Adalimumab phase III studies have shown that complete discontinuation of biologic treatment in patients with psoriasis who are in remission is not recommendable. Papp et al. showed that after the discontinuation of adalimumab treatment, $69 \%$ (178/256) of psoriasis patients showed a relapse after a median of five months (8). Similarly, Ortonne et al. demonstrated a time to relapse of only two months in 240/359 psoriasis patients in whom etanercept was discontinued (9). Complete discontinuation in ustekinumab-treated patients was also unsuccessful, $60 \%$ of patients had recurrence of disease activity after the median time of 15 weeks (10). Therefore, tapering of biologics appears to be a better option than complete discontinuation.

In Europe, off-label dosing of biologics is quite common in daily clinical practice $(5,11)$. This usually means not only dose escalation (e.g., doubling the dose), but also dose reductions, primarily by dosing interval prolongation. In daily practice, intensified dosing regimens are often applied in the early treatment phase and then continued as maintenance treatment, even when patients have been in remission for quite some time (1214). Disadvantages of unnecessary or excessive long-term dosing are not only the potentially higher risk of treatment-related side effects, such as serious infections and development of malignancies, but also the high costs that are associated with the use of biologic drugs $(15,16)$. In Europe, pharmaceutical companies have negotiated prices as high as 15,000 Euro per patient annually (17). As a result of the large numbers of patients now being treated with biologics, the pharmaceutical costs are a substantial proportion of the total health care budget. At present, evidence of successful prolongation of the per label dosing interval of biologics in psoriasis is scarce. Therefore, our primary objective was to determine the proportion of patients that could maintain a successful prolongation of the per label dosing interval. Secondary objectives were to evaluate the predictive value of baseline trough concentrations for successful dosing interval prolongation. In addition, we 
explored the feasibility of dosing interval prolongations in off-label-treated patients that were treated with either shorter of longer intervals according to the treatment label.

\section{Methods}

\section{Study design}

This was a prospective exploratory cohort study conducted at the department of Dermatology in the Erasmus Medical Center, Rotterdam, The Netherlands, between June 2013 and March 2016. The study was approved by the Institutional Review Board of the Erasmus University Medical Center Rotterdam (MEC-2013-050). The study was conducted according to the guidelines of Good Clinical Practice. The trial is registered in the European Clinical Trials Database (EudraCT) under EudraCT number 2012005809-53.

\section{Patients and treatments}

All included patients were 18 years or older and received maintenance treatment with adalimumab, etanercept, or ustekinumab for at least six months. In order to be eligible for the study, patients were required to have an absolute Psoriasis Activity and Severity Index (PASI) score below 8. Patients who used other anti-psoriatic co-medication such as methotrexate, fumarates, or acitretin were excluded; pregnant or lactating women were also excluded. All patients provided written informed consent before any of the study procedures were executed. Patients were recruited from the dermatology outpatient clinic of our hospital.

"Per label use" was defined as the use of the biologic for the approved indication, in the approved dosage and route of administration. In our study, "off-label use" implies that the dose or dose interval of either of the three biologics used in this study was not according to the package insert approved by our national drug regulatory agency. 


\section{Study procedures}

All patients were on maintenance treatment with adalimumab, etanercept, or ustekinumab with a dose and dosing interval prescribed by their dermatologist. Patient visits were scheduled at weeks $0,6,18,30$, $42,54,66$, and 78. At each study visit, data were collected on biologic trough concentration and PASI scores, adverse events, and laboratory testing (full blood count, aspartate aminotransferase, alanine aminotransferase, bilirubin, gamma-GT, serum creatinine, sedimentation rate, C-reactive protein, and urine analysis). The drug adherence was self-monitored, and empty syringes were collected during the outpatient visits. To determine whether a patient had stable disease activity, a run-in period of six weeks was used before the start of the study where the PASI score of the patient was not allowed to fluctuate more than three points. In all patients, the dosing interval of the biologic was prolonged every 12 weeks during a 42-week period (see supplementary table 1 for predefined schedule). In case of an unacceptable increase in disease activity judged by the patient or a rise of the PASI score above 8 , the last effective treatment regimen was resumed and was maintained during the remainder of the study period. Patients were followed up to week 78 .

Patients in whom the dosing interval could be prolonged according to the protocol without loss of selfreported efficacy or deterioration of the PASI score above 8 were designated "successful." Patients in whom the dosing interval could not be prolonged according to our protocol were designated "failure. "Failure" also included patients who objected to continuing with the dosing interval prolongation because of (self and investigator reported) exacerbation of their psoriasis or for other (personal) reasons. In the majority of patients, tapering consisted of dosing interval extensions (supplementary table 1 http://links.lww.com/TDM/A189) and in only a minority as dose reductions (with unchanged interval). In this paper, tapering will only be referred to as dosing interval prolongations. 


\section{Psoriasis Activity and Severity Index (PASI) and Physician Global Assessment (PGA)}

The Psoriasis Activity and Severity Index (PASI) is a validated tool for monitoring the disease activity.

The PASI is calculated based on the intensity of redness, thickness, scaling, and the affected surface area of the skin (18). The PASI score is the main outcome measure for success of treatment in clinical practice and clinical trials. The 5-point PGA scale is a modified and more simplified tool for evaluating plaque psoriasis severity and improvement in clinical trials. It is divided in clear, almost clear, mild, moderate, and severe score for the disease activity (19).

\section{Dermatology Life Quality Index (DLQI)}

It is a 10-item questionnaire used to measure the impact of a skin disease on the quality of life of an affected person. Each question refers to the impact of the skin disease on the patient's life over the previous week. The DLQI can provide more insight into the impairment of quality of life of psoriasis patients and can support more appropriate clinical decisions.

\section{Biologic assay}

Anti-TNFa biologic (adalimumab and etanercept) concentration was determined by an ELISA-based assay of Sanquin (Sanquin Reagents, Sanquin, Amsterdam, The Netherlands), according to manufacturer's instructions. Briefly, ELISA plates pre-coated with TNFa-specific murine monoclonal antibody (CLB-TNF5, Sanquin) were incubated with recombinant TNFa, followed by biologic containing calibrators, controls, and samples, diluted in HPE (High Performance ELISA) buffer (Sanquin). Binding of anti-TNFa biologic was detected with a horse-radish peroxidase-labeled monoclonal antibody specific for the adalimumab or etanercept idiotype. Both assays were in-house validated by Sanquin (Diagnostic 
Services, Biologics Lab). The adalimumab assay is a CE-IVD kit of Sanquin Reagents B.V. (http://www.sanquin.nl/repository/reagentia/ifu/M2910 2 level-adalimumab en.pdf), whereas the etanercept assay is a research assay and has no CE-IVD label. The adalimumab assay had $99 \%$ accuracy at $2 \mu \mathrm{g} / \mathrm{mL}$ and etanercept had $94 \%$ accuracy at $1 \mu \mathrm{g} / \mathrm{mL}$ of a spiked human serum sample. The lower limit of detection was 0.06 and $0.15 \mu \mathrm{g} / \mathrm{mL}$ for adalimumab and etanercept, respectively. The lower limit of quantification was 0.06 and $0.18 \mu \mathrm{g} / \mathrm{mL}$ for adalimumab and etanercept, respectively; the calibrator range was $0-25$ and $0-289 \mathrm{ng} / \mathrm{mL}$ for adalimumab and etanercept, respectively. The manual is available at http://www.sanquin.nl/en/products-services/reagents/product-categories/biologicals/. Day-to-day imprecision of these assays was $10.0 \%$ and $5.6 \%$, performed in 20 days for adalimumab concentration, and was $11.3 \%$ and $8.1 \%$, performed in 19 days for etanercept concentration, based on repetitive testing of low and high controls. For ustekinumab measurements, an analogous ELISA-based assay was used; however, this time, it was executed by Sanquin (Diagnostic Services, Biologicals Lab). In this in-house validated assay, plates were pre-coated with one of the ustekinumab targets, interleukin-12, to capture ustekinumab, and, for detection, rabbit polyclonal antibody specific for ustekinumab idiotype was used for detection (20). For ustekinumab PK measurements prior to Feb 2017, we gathered the following data: accuracy was $97 \%$ at $0.4 \mu \mathrm{g} / \mathrm{mL}$ of spiked human serum sample; lower limit of detection was $0.02 \mathrm{ng} / \mathrm{mL}$; lower limit of quantification was $0.02 \mu \mathrm{g} / \mathrm{mL}$; and the calibrator range was $0.0004-0.025 \mu \mathrm{g} / \mathrm{mL}$. The reportable range was $0.02-16 \mu \mathrm{g} / \mathrm{mL}$. Day-to-day imprecision of the ustekinumab assay was $9 \%$, based on three days of repetitive testing of a single (medium) control.

Measurement of anti-drug antibody against a biologic (ADA) was only performed in patients with undetectable biologic trough levels, as it is more likely to find ADA in absence of detectable drug (21). ADA was also determined by Sanquin (Diagnostic Services, Biologicals Lab), using in-house-validated ELISA-based assays. Etanercept has no clinically relevant immunogenity, and therefore measurement of ADA was not indicated (22). 


\section{Statistical analysis}

For numeric variables that were not normally distributed according to the Shapiro-Wilk test, we used the Mann Whitney U-test to compare groups. For the analysis of the associations between categorical data, we used Pearson's Chi Square test or Fisher's exact test, as appropriate. The difference between the biologic baseline trough concentration of the successes and the failures was analyzed with the Mann Whitney U-test. Statistical significance was defined as a two-tailed p value of 0.05 or less. For statistical data analysis, SPSS statistics software (version 23.0., IBM, Armonk, NY) was used.

\section{Results}

\section{Patients}

This study started in June 2013, and the last patient completed the follow-up in March 2016. In total, 64 patients were enrolled, and after the run-in period of six weeks, 59 patients were included (Fig. 1). Patient demographics and baseline disease characteristics are presented in Table 1. The BMI, PASI, PGA, and DLQI were significantly higher in the ustekinumab group compared with the adalimumab and etanercept group.

Seventeen out of 20 patients on adalimumab therapy (85\%) completed the 78-week study, 18 of 21 patients $(86 \%)$ in the etanercept therapy group completed the study, and 17 of 18 patients $(94 \%)$ in the ustekinumab therapy completed the study. Seven patients did not complete the study because of withdrawal of consent $(n=3)$ or because they were lost to follow up $(n=4)$ because of migration (moved to a different city or country). In each biologic group, patients had different maintenance dosing intervals, but the main cohort started with the per label dose and dosing interval (Fig. 1). The per label dosing interval for adalimumab was $40 \mathrm{mg}$ once every two weeks, for etanercept $50 \mathrm{mg}$ every week, and for ustekinumab $45 \mathrm{mg}(<100 \mathrm{~kg}$ ) or $90 \mathrm{mg}$ ( $>100 \mathrm{~kg}$ ) every 12 weeks. For etanercept and adalimumab, the dose was unchanged during the study protocol, only the dose interval was prolonged step by step. In 
ustekinumab-treated patients, the dose was also unchanged, except in three patients, in whom the dose was reduced from 90 to $45 \mathrm{mg}$.

\section{Prolongation of the dosing interval}

\section{Per label dosing}

At week 78, in the adalimumab group (40 mg every two weeks), seven out of 16 (44\%) patients had achieved a successful dosing interval prolongation. Six of these seven patients had a successful interval prolongation of $100 \%$ (40 mg every four weeks) and one of 50\% (40 mg every three weeks). In the etanercept group (50 mg every week), 5 out of $16(31 \%)$ patients had a successful dosing interval prolongation of 100\% (50 mg every two weeks).

In the ustekinumab group (45 mg every 12 weeks), two out of nine (22\%) patients had a successful dosing interval prolongation of $100 \%$ ( $45 \mathrm{mg}$ every 24 weeks).

\section{Off-label dosing}

The dosing interval could not be extended for the patients who were already being treated with adalimumab $40 \mathrm{mg}$ every three weeks at baseline $(n=4)$. In all four patients, the severity of psoriasis increased after dosing interval prolongation, and patients requested to be treated with their original regimen of adalimumab $40 \mathrm{mg}$ every three weeks.

In the escalated dose group, etanercept $50 \mathrm{mg}$ twice a week, one out of five (20\%) patients had a successful interval prolongation of $100 \%$ (50 mg once a week; per label dosing interval).

In the escalated dose group (ustekinumab $45 \mathrm{mg}$ every eight weeks), one out of three (33\%) patients was successfully prolonged to $45 \mathrm{mg}$ every 14 weeks. There was only one patient on ustekinumab $45 \mathrm{mg}$ every 10 weeks. In this patient, the dosing interval was successfully prolonged to 45 mg every 12 weeks ((Fig. 1). In the other off-label treated patients dosing interval prolongations were unsuccessful. 


\section{Trough concentrations}

The median baseline trough concentration of adalimumab in patients in whom the dosing interval was successfully prolonged was higher compared to the concentrations in the failures, although the difference was not statistically different $(8.8 \mu \mathrm{g} / \mathrm{mL}, 95 \%$ CI $[5.2 ; 10.6]$ vs. $5.3 \mu \mathrm{g} / \mathrm{mL}, 95 \%$ CI $[4,5 ; 10,9] \mathrm{p}=0.37)$ (Fig. 3A). The median baseline trough concentration of etanercept did not differ significantly between the successful prolongations vs. failures $(4.1 \mu \mathrm{g} / \mathrm{mL}, 95 \%$ CI $[0.0 ; 4.7]$ vs. $3.6 \mu \mathrm{g} / \mathrm{mL}, 95 \%$ CI $[1.3 ; 6.9], \mathrm{p}=1.0)$ (Fig. 3b). Also, the median baseline trough concentration of ustekinumab did not differ significantly between the successful prolongations vs. failures $(0.6 \mu \mathrm{g} / \mathrm{mL}, 95 \% \mathrm{CI}[0.3 ; 0.9]$ vs. $0.2 \mu \mathrm{g} / \mathrm{mL}, 95 \% \mathrm{CI}[0,2 ; 0,5] \mathrm{p}=$ 0,33) (Fig. 3c).

\section{Undetectable trough concentrations and the presence of ADA}

In the adalimumab group, three patients had, on two occasions, undetectable concentrations. In one of these patients, the presence of ADA was detected once; for this patient, psoriasis was in remission. In the etanercept group, two patients had, each on two occasions, undetectable concentrations and four patients showed an undetectable concentration at a single occasion. In total, 9 patients had 14 occasions in which undetectable trough concentrations were found. In the ustekinumab group, all patients had detectable trough concentrations, and the measurement of ADA was therefore not indicated.

\section{Loss of efficacy}

In the majority $(\mathrm{n}=55)$ of patients, the PASI score remained below 8 . In one patient in the per label adalimumab group and in two ustekinumab-treated patients on off-label escalated dose, the prolongation of the dosing interval caused an increase in disease activity that led to a PASI above 8. In one patient on offlabel escalated etanercept dose, the PASI increased above 8 before any interval prolongation, and in these cases, a dose prolongation was not performed. All of the four patients mentioned in this paragraph reached 
remission after they were retreated according to their original dosing regimen.

\section{Occurrence of adverse events (AE)}

The most frequent minor adverse events were respiratory and urinary tract infections. In the per label adalimumab group, three severe adverse events (SAE) occurred. One patient developed meningitis, and his psoriasis exacerbated. His PASI score reached 7.6, and at the end of the study, he was switched from adalimumab to ustekinumab. The other two patients suffered from rhabdomyolysis and had a coronary artery stent placement, respectively. In the per label ustekinumab group, two SAEs occurred (cerebrovascular accident and miscarriage). In the off-label group, one patient on ustekinumab was admitted to the hospital with myocardial infarction. All patients recovered without sequelae.

\section{Discussion}

The main finding of this study is that in approximately $30 \%$ of psoriasis patients on maintenance treatment with adalimumab, etanercept, or ustekinumab, the per label dosing interval could be prolonged without loss of clinical response.

The adalimumab group had the highest percentage of patients (44\%) in whom the dosing interval could be prolonged successfully. Baniandres et al. also showed that the adalimumab dose could be successfully reduced in $57.7 \%$ of patients in a cross-sectional study among 112 psoriasis patients (5). Rodrigo-Nicolas et al. showed 75\% successful adalimumab dosing interval prolongations in 12 patients (4). Their follow-up time of 46.5 weeks was shorter than our follow-up time of 78 weeks. In our study, $50 \%$ (three out of six) of the failures occurred after a follow-up of 52 weeks, potentially explaining the higher success rate of RodrigoNicolas et al.

In the ustekinumab per label group, a low rate (22\%) of successful interval prolongation was achieved. The ustekinumab-treated patients had a significantly higher PASI and DLQI score at baseline and they had previously been treated with significantly more biologics before they started using ustekinumab. Possibly the lower success rate in the ustekinumab group was related to more treatment-resistant disease. 
Trough concentrations of etanercept and ustekinumab at baseline did not correlate with the success of subsequent dosing interval prolongations. Concentration-effect relationships have not been shown for these two biologics. Neither Mahil et al. nor Menting et al. found a correlation between etanercept or ustekinumab trough concentrations and clinical response in newly treated psoriasis patients $(20,23,24)$. Van Herwaarden et al. also showed that adalimumab and etanercept trough concentrations were not predictive for successful dose reductions (24). In the adalimumab group of our study cohort, there was a trend toward higher trough concentrations in the patients in whom dosing interval could be prolonged, although the difference was not statistically significant. Our results support the finding of Menting et al. who showed that adalimumab concentrations above $7 \mu \mathrm{g} / \mathrm{mL}$ do not provide additional clinical efficacy (7). The majority (71\%) of adalimumab-treated patients in whom the dosing interval could be prolonged had an adalimumab trough concentration above $7 \mu \mathrm{g} / \mathrm{mL}$ at baseline.

Another important secondary aim of our study was the safety of dosing interval prolongations. Reported risk of biologic dose reductions such as irreversible loss of efficacy and immunogenicity (25) appeared to be low/absent in this study. Dose reductions have a major influence on the costs of treatment, if patients can be treated with considerably less injections per year because the dosing interval is doubled. Potentially, this can result in treatment cost savings of 7,021 euros per adalimumab-treated patient; 6,939 euros per etanercepttreated patient; and 7,130 euros per ustekinumab-treated patient per year. Indirect costs should also be taken into account for a formal and more accurate cost-effective evaluation.; However, this was beyond the scope of our study. Some studies in rheumatoid arthritis patients on treatment with biologics have published promising data on major savings in the costs for biologics after dose reduction $(26,27)$.

In clinical practice, patients are often treated off-label; therefore, we also included these patients in our study. Successful prolongations were less often observed in the off-label group. In the off-label adalimumab and ustekinumab group that was already on a prolonged dosing interval, further dosing interval prolongation was unsuccessful.

A limitation of this exploratory study was the relatively small number of patients per biologic. A control group of patients who continued maintenance treatment without dose interval prolongation was not a part 
of the current study design. For larger prospective trials, inclusion of a control group will help to estimate the incidence of psoriasis flares, in patients treated with unchanged dose and dose interval. Moreover, we chose a relatively high PASI of 8 as a cutoff for inclusion in this study and to define the maximum allowed deterioration in disease activity upon dosing interval prolongation. In retrospect, a PASI of 5 would have been more appropriate since this has recently been suggested as a cutoff for intervention (28). However, the higher PASI cutoff does not appear to have influenced our results in this light, since all included patients had low disease activity with the highest PASI at baseline of 4.8 (etanercept group). Furthermore, most of the patients that experienced deterioration of their psoriasis upon dosing interval prolongation chose to return to their previous dosing interval before a PASI of 5 was reached. Also, none of the patients that were designated as "successful" upon dosing interval prolongations had a PASI above 5. Despite these limitations, this is one the first observational biologic tapering studies reflecting a real-life setting in a clinical dermatology practice. A definition of clinical criteria to select patients in whom dose interval prolongation can be applied would be helpful. Intuitively one would think that a longer duration of remission, no failed biologic treatments prior to the current treatment, a very low PASI score, and higher biologic concentrations would increase the chance of successful dose interval prolongation. Larger cohorts of patients are needed to evaluate the predictive value of the mentioned clinical criteria.

In conclusion, safe and robust prolongation of biologic dosing intervals was achieved in almost $30 \%$ of patients. This can reduce the treatment costs without clinically relevant loss of efficacy. Larger (costeffectiveness) studies are warranted to corroborate our findings and to investigate whether adalimumab baseline trough levels can indeed predict the successful interval prolongation in psoriasis patients with already stable mild disease. Moreover, these investigations could possibly be extended to the newly introduced anti-IL17 biologics for which the data on safe dosing interval prolongations in psoriasis are also lacking. 


\section{References}

1. Den Broeder AA, Creemers MC, van Gestel AM, et al. Dose titration using the Disease Activity Score (DAS28) in rheumatoid arthritis patients treated with anti-TNF-alpha.

Rheumatology (Oxford). 2002;41:638-642.

2. van der Maas A, Kievit W, van den Bemt BJ, et al. Down-titration and discontinuation of infliximab in rheumatoid arthritis patients with stable low disease activity and stable treatment: an observational cohort study. Ann Rheum Dis. 2012;71:1849-1854.

3. Chen DY, Chen YM, Hsieh TY, et al. Drug trough levels predict therapeutic responses to dose reduction of adalimumab for rheumatoid arthritis patients during 24 weeks of follow-up. Rheumatology (Oxford). 2016;55:143-148.

4. Rodrigo-Nicolas B, Gimeno-Carpio E, Quecedo-Estebanez E, et al. Adalimumab dose reduction in psoriasis: Results in a series of 12 patients. Journal of the American Academy of Dermatology.70(5):AB164.

5. Baniandres O, Rodriguez-Soria VJ, Romero-Jimenez RM, et al. Dose modification in biologic therapy for moderate to severe psoriasis: a descriptive analysis in a clinical practice setting. Actas Dermosifiliogr. 2015;106:569-577.

6. Pouw MF, Krieckaert CL, Nurmohamed MT, et al. Key findings towards optimising adalimumab treatment: the concentration-effect curve. Ann Rheum Dis. 2015;74:513-518.

7. Menting SP, Coussens E, Pouw MF, et al. Developing a therapeutic range of adalimumab serum concentrations in management of psoriasis: a step toward personalized treatment. JAMA Dermatol. 2015;151:616-622.

8. Papp K, Crowley J, Ortonne JP, et al. Adalimumab for moderate to severe chronic plaque psoriasis: efficacy and safety of retreatment and disease recurrence following withdrawal from therapy. Br J Dermatol. 2011;164:434-441.

9. Ortonne JP, Taieb A, Ormerod AD, et al. Patients with moderate-to-severe psoriasis recapture clinical response during re-treatment with etanercept. $\mathrm{Br} J$ Dermatol. 2009;161:11901195.

10. Leonardi CL, Kimball AB, Papp KA, et al. Efficacy and safety of ustekinumab, a human interleukin-12/23 monoclonal antibody, in patients with psoriasis: 76 -week results from a randomised, double-blind, placebo-controlled trial (PHOENIX 1). Lancet. 2008;371:1665-1674. 11. Carrascosa JM, Garcia-Doval I, Perez-Zafrilla B, et al. Use of off-label doses is frequent in biologic therapy for moderate to severe psoriasis: a cross-sectional study in clinical practice. $J$ Dermatolog Treat. 2015;26:502-506.

12. Moots RJ, Haraoui B, Matucci-Cerinic M, et al. Differences in biologic dose-escalation, nonbiologic and steroid intensification among three anti-TNF agents: evidence from clinical practice. Clin Exp Rheumatol. 2011;29:26-34.

13. Mrowietz U, Kragballe K, Reich K, et al. Definition of treatment goals for moderate to severe psoriasis: a European consensus. Arch Dermatol Res. 2011;303:1-10.

14. Bultman E, de Haar C, van Liere-Baron A, et al. Predictors of dose escalation of adalimumab in a prospective cohort of Crohn's disease patients. Aliment Pharmacol Ther. 2012;35:335-341.

15. Bongartz T, Sutton AJ, Sweeting MJ, et al. Anti-TNF antibody therapy in rheumatoid arthritis and the risk of serious infections and malignancies: systematic review and meta-analysis of rare harmful effects in randomized controlled trials. JAMA. 2006;295:2275-2285.

16. Singh JA, Christensen R, Wells GA, et al. Biologics for rheumatoid arthritis: an overview of Cochrane reviews. Cochrane Database Syst Rev. 2009:CD007848.

17. Gelfand JM, Weinstein R, Porter SB, et al. Prevalence and treatment of psoriasis in the United Kingdom: a population-based study. Arch Dermatol. 2005;141:1537-1541.

18. Feldman SR, Krueger GG. Psoriasis assessment tools in clinical trials. Ann Rheum Dis. 2005;64:ii65-8; discussion ii9-73. 
19. Langley RG, Feldman SR, Nyirady J, et al. The 5-point Investigator's Global Assessment (IGA) Scale: a modified tool for evaluating plaque psoriasis severity in clinical trials. $J$ Dermatolog Treat. 2015;26:23-31.

20. Menting SP, van den Reek JM, Baerveldt EM, et al. The correlation of clinical efficacy, serum trough levels and antidrug antibodies in ustekinumab-treated patients with psoriasis in a clinical-practice setting. Br J Dermatol. 2015;173:855-857.

21. Rispens $T$, Hart MH, Ooijevaar-de Heer $P$, et al. Drug interference in immunogenicity assays depends on valency. J Pharm Biomed Anal. 2013;85:179-185.

22. de Vries MK, van der Horst-Bruinsma IE, Nurmohamed MT, et al. Immunogenicity does not influence treatment with etanercept in patients with ankylosing spondylitis. Ann Rheum Dis. 2009;68:531-535.

23. Mahil SK, Arkir Z, Richards G, et al. Predicting treatment response in psoriasis using serum levels of adalimumab and etanercept: a single-centre, cohort study. $\mathrm{Br} J$ Dermatol. 2013;169:306-313.

24. van Herwaarden N, Bouman CA, van der Maas A, et al. Adalimumab and etanercept serum (anti)drug levels are not predictive for successful dose reduction or discontinuation in rheumatoid arthritis. Ann Rheum Dis. 2015;74:2260-2261.

25. Chan CK, Holroyd CR, Mason A, et al. Are there dangers in biologic dose reduction strategies? Autoimmun Rev. 2016;15:742-746.

26. Murphy CL, Awan S, Sullivan MO, et al. Major cost savings associated with biologic dose reduction in patients with inflammatory arthritis. Ir Med J. 2015;108:19-21.

27. Raffeiner B, Botsios C, Ometto F, et al. Effects of half dose etanercept (25 mg once a week) on clinical remission and radiographic progression in patients with rheumatoid arthritis in clinical remission achieved with standard dose. Clin Exp Rheumatol. 2015;33:63-68.

28. Dauden E, Puig L, Ferrandiz C, et al. Consensus document on the evaluation and treatment of moderate-to-severe psoriasis: Psoriasis Group of the Spanish Academy of Dermatology and Venereology. J Eur Acad Dermatol Venereol. 2016;30:1-18.

\section{Figure legends}

Fig. 1: Study flow chart showing patients who are included in the study per biologic and dosing interval

groups. The number of patients with successful prolongation of the dosing interval and failures is

depicted. Patients who did not complete the study are also shown for each group.

Fig. 2a: Comparison of adalimumab trough concentrations at baseline after the run-in period of six weeks in patients with a successful dosing interval prolongation and the failures. The concentrations at baseline of each patient are represented by a circle (०). The horizontal line in the graph represents the median.

Fig. 2b: Comparison of etanercept trough concentrations at baseline after the run-in period of six weeks in patients with a successful dosing interval prolongation and failures. The concentrations at baseline of each patient are represented by a circle (०). The horizontal line in the graph represents the median. 
Fig. 2c: Comparison of ustekinumab trough concentrations at baseline after the run-in period of six weeks in patients with a successful dosing interval prolongation and failures. The concentrations at baseline of each patient are represented by a circle (०). The horizontal line in the graph represents the median.

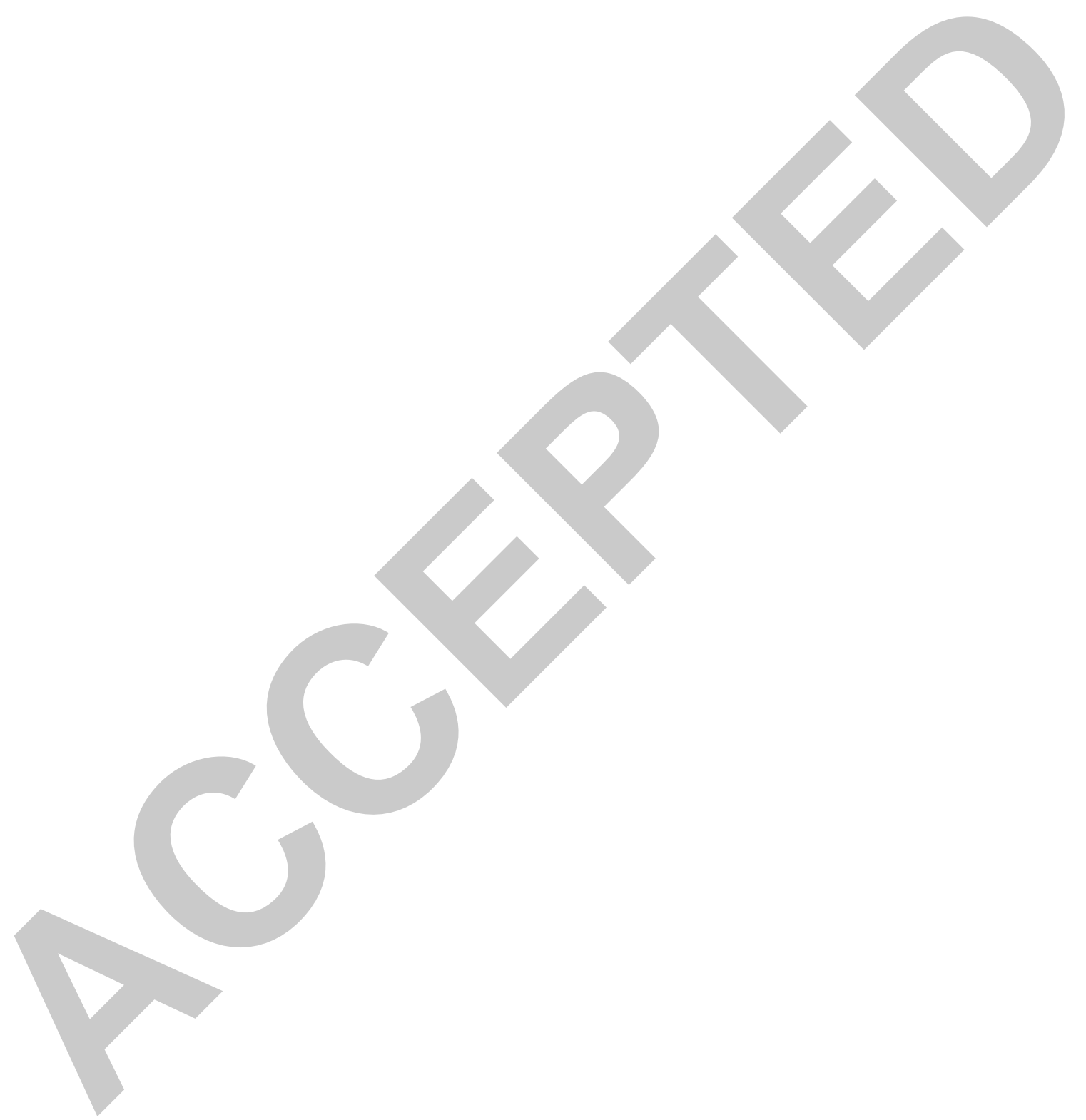


Table 1 Baseline patient characteristics.

\begin{tabular}{|c|c|c|c|}
\hline $\begin{array}{l}\text { Patient } \\
\text { characteristics }\end{array}$ & $\begin{array}{l}\text { Adalimumab }(\mathrm{n}=20) \\
\mathrm{n}(\%)\end{array}$ & $\begin{array}{l}\text { Etanercept }(n=21) \\
n(\%)\end{array}$ & $\begin{array}{l}\text { Ustekinumab }(\mathrm{n}=18) \\
\mathrm{n}(\%)\end{array}$ \\
\hline $\begin{array}{l}\text { Gender, n (\%) } \\
\text { Males } \\
\text { Females }\end{array}$ & $\begin{array}{r}16(80) \\
4(20)\end{array}$ & $\begin{array}{r}12(57) \\
9(43)\end{array}$ & $\begin{array}{r}11(61) \\
7(39)\end{array}$ \\
\hline $\begin{array}{l}\text { Race, n (\%) } \\
\text { Caucasian } \\
\text { Black } \\
\text { Asian } \\
\text { Other }\end{array}$ & $\begin{array}{r}13(65) \\
1(5) \\
5(25) \\
1(5)\end{array}$ & $\begin{array}{l}15(71) \\
4(19) \\
2(10)\end{array}$ & $\begin{array}{l}14(78) \\
2(11) \\
2(11)\end{array}$ \\
\hline $\begin{array}{l}\text { Age (years), mean } \\
\text { (SD) }\end{array}$ & $43.2(13.2)$ & $50.6(14.4)$ & $52.0(15.3)$ \\
\hline $\begin{array}{l}\text { Body mass index } \\
(\mathrm{kg} / \mathrm{m} 2), \text { median } \\
(\mathrm{Q} 1, \mathrm{Q} 3)\end{array}$ & $24(23,29)$ & $26(23,2)$ & $28(26,31)$ \\
\hline $\begin{array}{l}\text { PASI score, } \\
\text { Median (Q1, Q3) }\end{array}$ & $1.7(0.3,3.2)$ & $3.2(1.3,4.8)$ & $2.9(1.8,3.9)$ \\
\hline $\begin{array}{l}\text { PGA score } \\
\text { Clear } \\
\text { Almost clear } \\
\text { Mild }\end{array}$ & $\begin{array}{r}6(30) \\
14(70)\end{array}$ & $\begin{array}{r}4(19) \\
15(71)\end{array}$ & $\begin{array}{r}3(17) \\
13(72) \\
2(11)\end{array}$ \\
\hline $\begin{array}{l}\text { DLQI score, } \\
\text { Median (Q1, Q3) }\end{array}$ & $0.0(0.0,2.5)$ & $1.0(0.0,5.0)$ & $1.5(0.0,5.3)$ \\
\hline $\begin{array}{l}\text { Duration of } \\
\text { psoriasis, years, } \\
\text { Median (Q1, Q3) }\end{array}$ & $15(8,28)$ & $20(14,34)$ & $22(14,39)$ \\
\hline $\begin{array}{l}\text { History of psoriatic } \\
\text { arthritis, n (\%) }\end{array}$ & $5(25)$ & $2(10)$ & $2(11)$ \\
\hline $\begin{array}{l}\text { Biologic-naïve } \\
\text { before start current } \\
\text { biologic, } \mathrm{n}(\%)\end{array}$ & $12(60)$ & $15(71)$ & $4(22)$ \\
\hline
\end{tabular}


Figure 1

Included in study $(n=64)$

\{

Not eligible after run-in period $(n=2)$

Withdrew consent after run-in period $(n=3)$

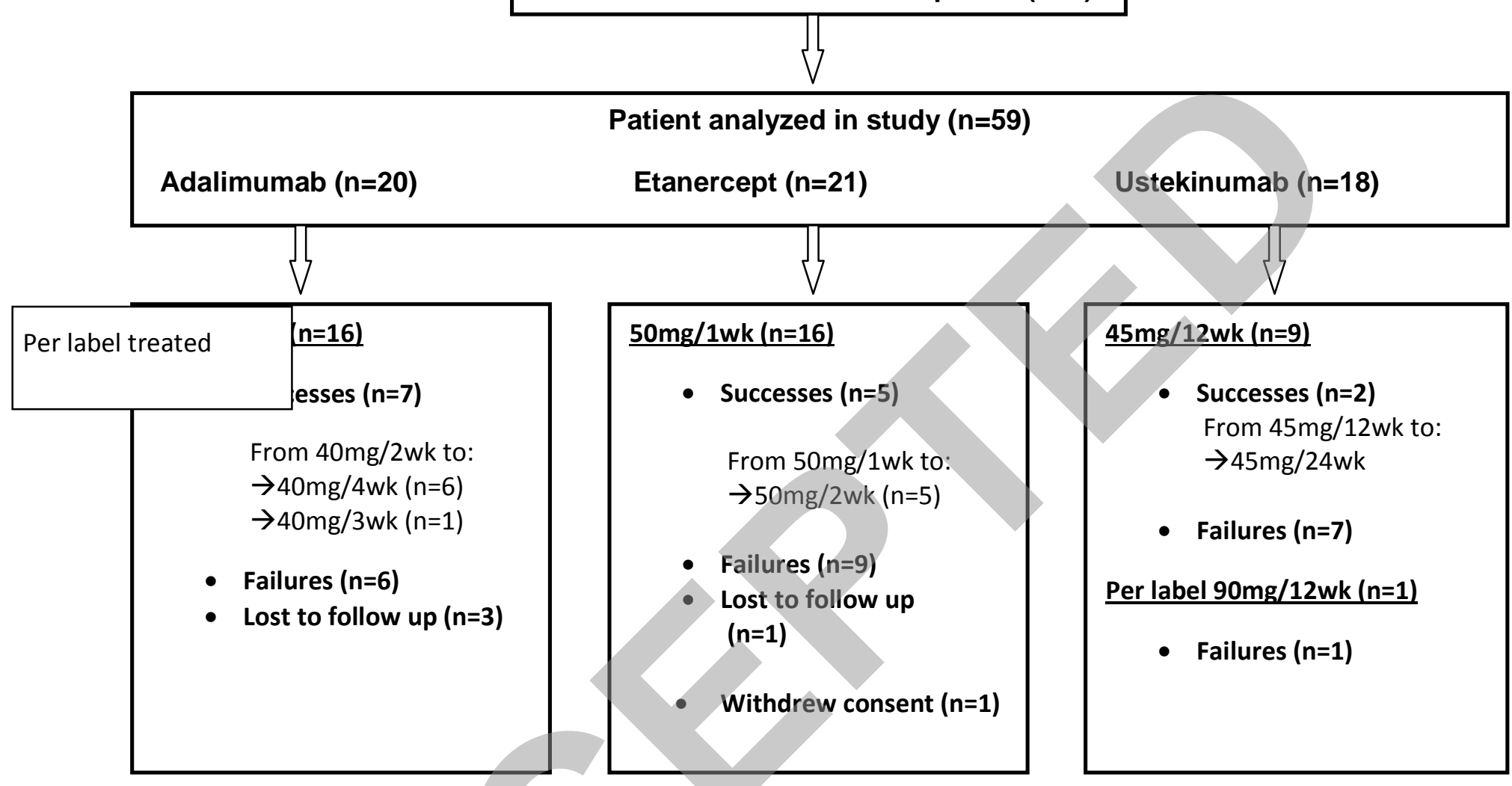

Off label treated

a=4)

- Failures $(n=4)$

\section{0mg $2 \times$ wk $(n=5)$}

- Successes $(n=1)$

From 50mg 2x wk to:

$\rightarrow 50 \mathrm{mg} / 1 \mathrm{wk}$

- Failures $(n=3)$

- Withdrew consent $(n=1)$
$45 \mathrm{mg} / 8 \mathrm{wk}(\mathrm{n}=3)$

- Successes $(n=1)$ From $45 \mathrm{mg} / 8 \mathrm{wk}$ to $\rightarrow 45 \mathrm{mg} / 14 \mathrm{wk}$

- Failures $(n=2)$

$45 \mathrm{mg} / 10 \mathrm{wk}(\mathrm{n}=1)$

- Successes $(n=1)$ From $45 \mathrm{mg} / 10 \mathrm{wk}$ to $\rightarrow 45 \mathrm{mg} / 12 \mathrm{wk}$

$45 \mathrm{mg} / 16 \mathrm{wk}(\mathrm{n}=1)$

- Withdrew consent $(n=1)$

$90 \mathrm{mg} / 8 \mathrm{wk}(\mathrm{n}=2)$

- Failures $(\mathrm{n}=2)$

$90 \mathrm{mg} / 16 \mathrm{wk}(\mathrm{n}=1)$

- Failures $(n=1)$ 
Figure 2A

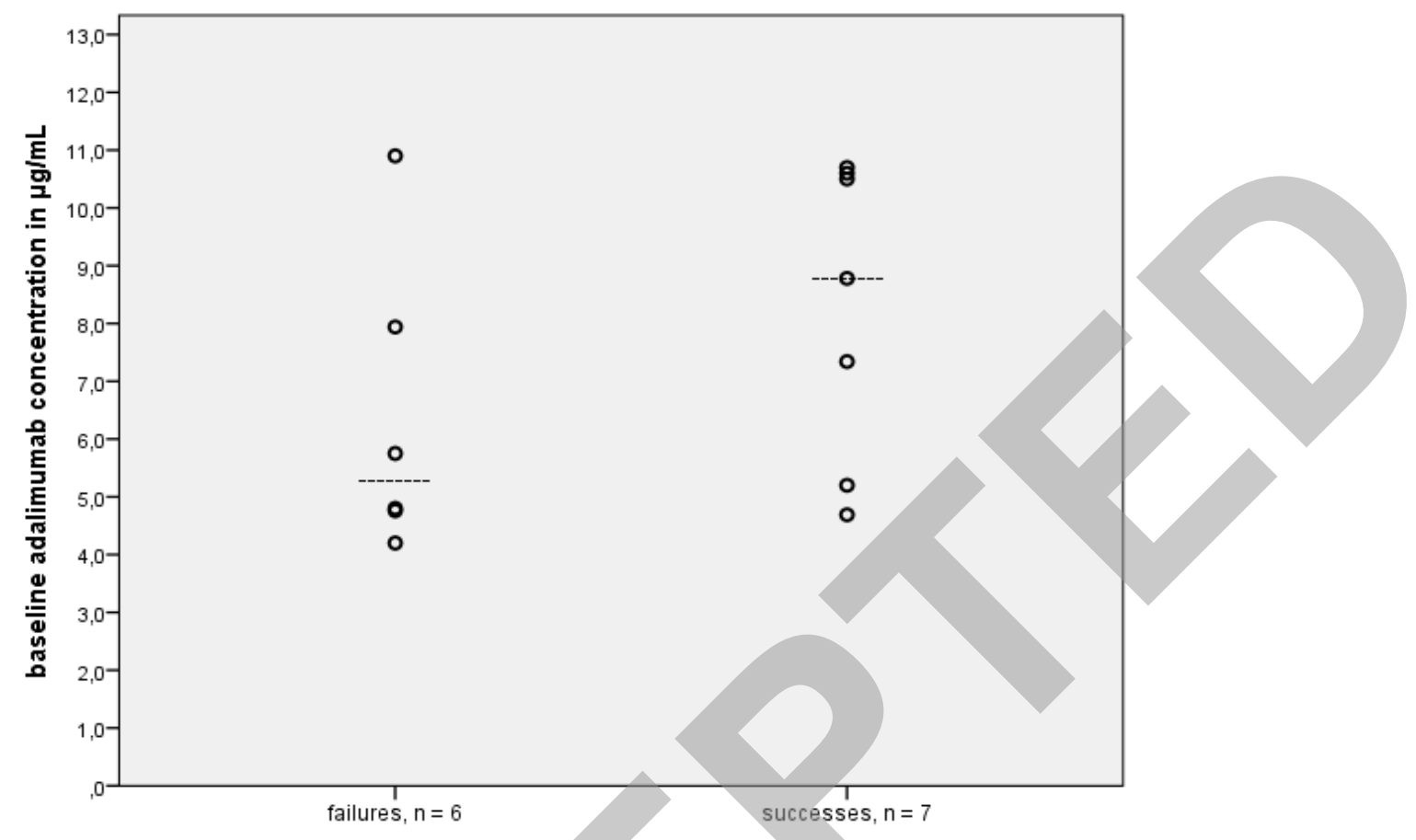

Figure 2B

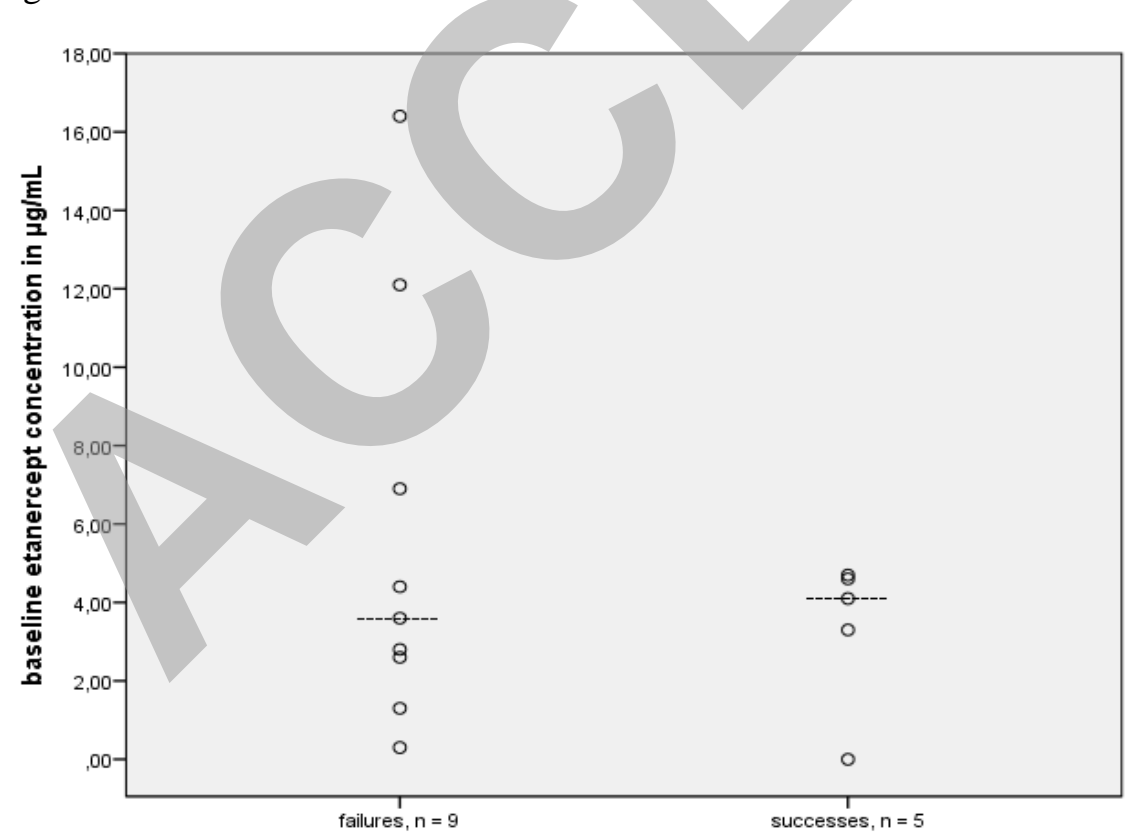


Figure 2C

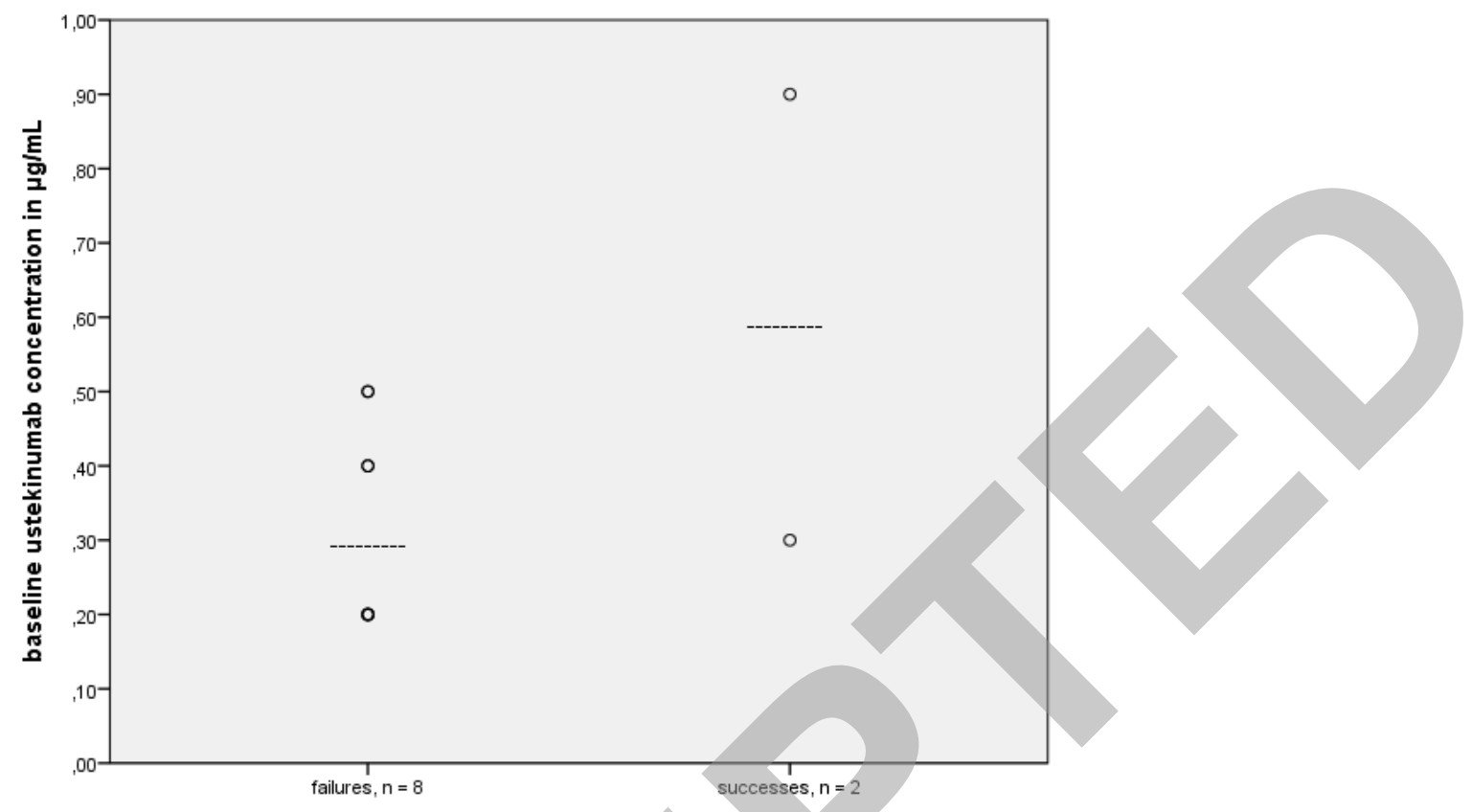

* Some circles (०) are on the same place in the graph because some patients have the same concentrations at baseline.

** The six patients who failed to complete the study were not included. 